\title{
Editorial Commentary: Tivozanib versus sorafenib in patients with advanced renal cell carcinoma (TIVO-3): a phase 3, multicentre, randomised, controlled, open-label study
}

\author{
Mary E. Westerman, Christopher G. Wood \\ Department of Urology, University of Texas MD Anderson Cancer Center, Houston, TX, USA \\ Correspondence to: Christopher G. Wood, MD. Douglas E. Johnson MD Professorship, Professor \& Deputy Chairman, Department of Urology, \\ University of Texas MD Anderson Cancer Center, 1515 Holcombe, Unit 1373, Houston, TX 77030, USA. Email: cgwood@mdanderson.org. \\ Provenance and Peer Review: This article was commissioned by the Editorial Office, Annals of Translational Medicine. The article did not undergo \\ external peer review. \\ Comment on: Rini BI, Pal SK, Escudier BJ, et al. Tivozanib versus sorafenib in patients with advanced renal cell carcinoma (TIVO-3): a phase 3, \\ multicentre, randomised, controlled, open-label study. Lancet Oncol 2020;21:95-104.
}

Submitted Mar 19, 2020. Accepted for publication Apr 24, 2020.

doi: 10.21037/atm.2020.03.217

View this article at: http://dx.doi.org/10.21037/atm.2020.03.217

In the January 2020 edition of The Lancet Oncology, Rini and colleagues reported the results of TIVO-3, an openlabel phase 3 randomized control trial (RCT) comparing tivozanib with sorafenib in patients with refractory metastatic clear cell renal cell carcinoma (mRCC) (1). Tivozanib is a highly selective vascular endothelial growth factor receptor (VEGFR) tyrosine kinase inhibitor (TKI) which inhibits phosphorylation of VEGFR1, VEGFR2, and VEGFR3 (2). It has a 4-5-day half-life, and ten-fold higher concentrations are required to inhibit cKIT and platelet derived growth factor (PDGF) (2). Compared to earlier generation TKIs, tivozanib was designed to optimize VEGFR blockade while minimizing off-target toxic effects, ultimately resulting in fewer dose interruptions and dose reductions $(2,3)$.

Initially, tivozanib was tested against sorafenib in the first-line setting for mRCC in TIVO-1, an international phase 3 trial (4). Tivozanib demonstrated significantly improved progression-free survival (PFS) compared to sorafenib (11.9 vs. 9.1 months; HR, 0.797; 95\% CI, 0.6390.993; $\mathrm{P}=0.04$ ), thus meeting its primary endpoint (4). However, there was a trend towards improved overall survival (OS) in the sorafenib arm (29.3 vs. 28.8 months; HR, 1.245; 95\% CI, 0.954-1.624; $\mathrm{P}=0.11$ ) (4). Given the improved PFS, tivozanib was approved in Europe for firstline treatment of mRCC, however because of concerns regarding the OS results, it was not approved in the United
Stated (5).

The OS results in TIVO-1 were confounded by geographically driven imbalanced cross-over in second-line treatment. A large proportion of participants were from Eastern Europe with limited access to targeted therapies (4). Under an extension protocol, those who progressed in the sorafenib arm were eligible to receive sponsor-provided tivozanib, while those in the tivozanib arm could only receive best available therapy excluding sorafenib (4). This resulted in $63 \%$ of sorafenib patients receiving second-line tivozanib while only $13 \%$ of patients in the tivozanib arm received any second-line therapy (4). Notably, subgroup analysis restricted to participants from North America and Europe demonstrated an OS of 35.9 months in the tivozanib arm compared to 31 months for sorafenib (HR, 0.503; 95\% CI, 0.174-1.451; $\mathrm{P}=0.195$ ) (4). In addition, patients given tivozanib after progression on sorafenib had a median PFS of 11.0 months and OS of 21.6 months from the start of tivozanib therapy, indicating activity in the second-line setting (6).

Given this potential efficacy in later lines of therapy, TIVO-3 was designed as an open-label phase 3 RCT comparing tivozanib to sorafenib in patients who had received at least two prior lines of therapy (1). Between May 2016 and August 2017, 350 patients were enrolled across 120 sites (1). Randomization was done by permutated blocks with incorporation of two stratification factors-IMDC 
risk group and type of previous therapy (1). The primary outcome was PFS as assessed by an independent radiology committee, with numerous secondary endpoints, including OS (1). The authors report a significant difference in PFS at 5.6 months in the tivozanib arm compared to 3.9 months in the sorafenib arm (HR, 0.73; 95\% CI, 0.56-0.94; $\mathrm{P}=0.016$ ). Interim OS, assessed 2 years after enrollment of the final patient (10 months after the final PFS analysis) and after 227 (65\%) of patients had died, was 16.4 months (95\% CI, 13.4-22.2) in the tivozanib arm and 19.7 months (95\% CI, 15.0-24.2) in the sorafenib arm (HR, 0.99; 95\% CI, 0.761.29; $\log$-rank $\mathrm{P}=0.95$ ) (1). A final OS analysis is expected to report in June 2020 (7).

In TIVO-3, better PFS outcomes were seen among favorable (HR, 0.46; 95\% CI, 0.25-0.85, P=0.01) and intermediate IMDC risk patients (HR, 0.69; 95\% CI, 0.49$0.95 ; \mathrm{P}=0.02$ ), indicating ongoing responsiveness to VEGFR inhibition (8). These findings are similar to the results from the AXIS trial, in which patients treated with axitinib demonstrated a PFS of 8.3 months (95\% CI, 6.7-9.2) compared to 5.7 months (95\% CI, 4.7-6.5) for sorafenib (HR, 0.656; 95\% CI, 0.552-0.779; one-sided $\mathrm{P}<0.0001$ ) (9). MSKCC favorable risk patients appeared to receive the most benefit (HR, 0.497; 95\% CI, 0.326-0.758), implying angiogenesis continued to contribute to tumorigenesis $(9,10)$. Notably, like tivozanib, axitinib is a later generation VEGFR TKI, and despite the improvement in PFS in AXIS, no OS benefit was identified $(9,11)$.

While it is not possible to draw treatment superiority/ inferiority inferences by directly comparing effect sizes across clinical trials, prior findings in refractory mRCC trials provide context for interpreting TIVO-3 results. METEOR and CheckMate-025 were large phase three RCTs which evaluated the efficacy of cabozantinib and nivolumab respectively in refractory mRCC populations $(12,13)$. In 2015 , both drugs demonstrated significant OS benefit compared to everolimus and were approved for second-line use $(12,13)$. In each study, approximately $30 \%$ of patients had received at least two prior VEGFR or anti-angiogenic agents [METEOR: $\mathrm{n}=194 / 658,(29 \%)$; CheckMate-025: $n=230 / 803,(29 \%)]$, comparable to the TIVO-3 cohort $(12,13)$.

In TIVO-3, $45 \%$ of patients $(n=159)$ received two prior VEGFR TKIs (1). In this group, median PFS was 5.5 months (95\% CI, 3.6-7.4) with tivozanib and 3.7 months (95\% CI, 3.6-3.9) with sorafenib (HR, 0.58; 95\% CI, 0.4-0.8; $\mathrm{P}=0.0032$ ) (1). In METEOR, median OS was 21.4 months (95\% CI, 18.7-not estimable) in the cabozantinib arm compared to 16.5 months (95\% CI, 14.7-18.8) in the everolimus arm (HR, 0.66; 95\% CI, $0.53-0.83 ; \mathrm{P}=0.00026)(12,14)$. Notably, while PFS was improved in the subgroup with two prior VEGFR agents (HR, 0.51; 95\% CI, 0.35-0.74), there was no OS difference (HR, 0.73; 95\% CI, 0.48-1.10) in the final results (14). In CheckMate-025, the median OS was 25 months with nivolumab and 19.6 months with everolimus (HR, 0.73; 95\% CI, 0.57-0.93; $\mathrm{P}<0.002)$ (13). On subgroup analysis, those who had received two or more prior therapies had a statistically significant improvement in OS when given nivolumab compared to everolimus (HR, 0.65; 95\% CI, $0.43-0.99)(13,15)$.

Thus, among patients who have received two prior VEGFR therapies, the clinical relevance of a 55-day improvement in PFS with tivozanib should be critically evaluated given that radiologic progression does not imply symptomatic progression and prior trials have shown OS improvements $(16,17)$. In patients treated with first-line targeted therapy, PFS at 3 and 6 months was found to be predictive of OS; however, PFS may not be a valid surrogate endpoint for OS in later lines of therapy (18). Because of concerns related to OS, the FDA requested that the new drug application (NDA) for tivozanib be held until the final OS results are available from TIVO-3 (19). The company has indicated it will withdraw its NDA if the final OS results in a HR above 1.00 (19).

The second subgroup which benefitted from tivozanib were those previously receiving a checkpoint inhibitor (ICI) and a VEGFR TKI (n=91, 26\%) (1). Those given tivozanib had a median PFS of 7.3 (95\% CI, 4.8-11.1) vs. 5.1 months (95\% CI, 3.2-7.4) with sorafenib (HR, 0.55; 95\% CI, 0.32-0.94; P=0.02) (1). Enrollment for TIVO-3 closed in 2017, therefore most patients likely received a VEGFR agent followed by nivolumab monotherapy (13). In 2020, front line combination therapy is standard of care and the optimal sequencing strategy for subsequent agents such a nivolumab is unknown (10). Conclusions about the utility of tivozanib following combination therapy cannot be inferred on the basis of TIVO-3 alone. Trials to evaluate sequencing strategies for advanced RCC are needed, although challenging given the rapidly evolving treatment paradigm.

Finally, much of the interest regarding tivozanib has been due to the specificity of the molecule for VEGFR1, VEGFR2, and VEGFR3, hypothesized to reduce offtarget toxicity resulting in fewer dose interruptions and dose reductions $(2,3)$. In a recent study of the real-world use of targeted therapies, Aspinall and colleagues found 
that among 220 men with newly diagnosed mRCC treated in the United States Veterans Affairs (VA) system between $2010-2014,62.3 \%$ of patients had one or more doses held or reduced, typically due to an adverse drug event (20). In TIVO-3, adverse events (AEs) were reported in $94 \%$ of the sorafenib group and $84 \%$ of the tivozanib group (1). Serious AEs occurred in $43.4 \%$ of the tivozanib group and $39.4 \%$ of the sorafenib group and were considered treatmentrelated in $11 \%$ and $10 \%$ respectively $(1,7)$. There were fewer dose interruptions and dose reductions due to $\mathrm{AE}$ in the tivozanib group compared to sorafenib group (48\% and $24 \%$ vs. $63 \%$ and $38 \%$, respectively) (1). However, because the study was not blinded the authors note there is possible bias in toxicity assessments, potentially resulting in more dose alterations in the sorafenib group (1). In addition, there was no quality of life assessment performed, thus drawing conclusions about patient quality of life or treatment preference is not feasible (1). Notably, in TIVO-1 health related quality of life was measured and no differences between tivozanib and sorafenib were seen (4).

Ultimately, it is hard to know where tivozanib fits in the current mRCC treatment landscape. Outside of clinical trials, Aspinall found that the average number of targeted therapies received by patients with newly diagnosed $\mathrm{mRCC}$ was 1.9 and the median time from therapy initiation to death was 13 months (20). This sobering real-world data is a reminder we have a long way to go in the treatment of patients with mRCC. Tivozanib may be beneficial to a subset of patients, but without better methods to identify those patients the overall results from TIVO-3 do little to move the needle.

\section{Acknowledgments}

Funding: None.

\section{Footnote}

Conflicts of Interest: Both authors have completed the ICMJE uniform disclosure form (available at http://dx.doi. org/10.21037/atm.2020.03.217). The authors have no conflicts of interest to declare.

Ethical Statement: The authors are accountable for all aspects of the work in ensuring that questions related to the accuracy or integrity of any part of the work are appropriately investigated and resolved.
Open Access Statement: This is an Open Access article distributed in accordance with the Creative Commons Attribution-NonCommercial-NoDerivs 4.0 International License (CC BY-NC-ND 4.0), which permits the noncommercial replication and distribution of the article with the strict proviso that no changes or edits are made and the original work is properly cited (including links to both the formal publication through the relevant DOI and the license). See: https://creativecommons.org/licenses/by-nc$\mathrm{nd} / 4.0 \%$.

\section{References}

1. Rini BI, Pal SK, Escudier BJ, et al. Tivozanib versus sorafenib in patients with advanced renal cell carcinoma (TIVO-3): a phase 3, multicentre, randomised, controlled, open-label study. Lancet Oncol 2020;21:95-104.

2. Nakamura K, Taguchi E, Miura T, et al. KRN951, a highly potent inhibitor of vascular endothelial growth factor receptor tyrosine kinases, has antitumor activities and affects functional vascular properties. Cancer Res 2006;66:9134-42.

3. Eskens FA, de Jonge MJ, Bhargava P, et al. Biologic and clinical activity of tivozanib (AV-951, KRN-951), a selective inhibitor of VEGF receptor-1, -2 , and -3 tyrosine kinases, in a 4-week-on, 2-week-off schedule in patients with advanced solid tumors. Clin Cancer Res 2011;17:7156-63.

4. Motzer RJ, Nosov D, Eisen T, et al. Tivozanib versus sorafenib as initial targeted therapy for patients with metastatic renal cell carcinoma: results from a phase III trial. J Clin Oncol 2013;31:3791-9.

5. Tivozanib for the treatment of advanced renal cell carcinoma. Available online: https://wayback. archive-it.org/7993/20170405223310/https:// www.fda.gov/downloads/AdvisoryCommittees/ CommitteesMeetingMaterials/Drugs/ OncologicDrugsAdvisoryCommittee/UCM351322.pdf

6. Molina AM, Hutson TE, Nosov D, et al. Efficacy of tivozanib treatment after sorafenib in patients with advanced renal cell carcinoma: crossover of a phase 3 study. Eur J Cancer 2018;94:87-94.

7. A study to compare tivozanib hydrochloride to sorafenib in subjects with refractory advanced RCC. Available online: https://clinicaltrials.gov/ct2/show/results/NCT02627963? term=tivozanib \&draw $=4 \&$ rank $=22$

8. McDermott DF, Huseni MA, Atkins MB, et al. Publisher Correction: Clinical activity and molecular correlates of 
response to atezolizumab alone or in combination with bevacizumab versus sunitinib in renal cell carcinoma. Nat Med 2018;24:1941.

9. Rini BI, Escudier B, Tomczak P, et al. Comparative effectiveness of axitinib versus sorafenib in advanced renal cell carcinoma (AXIS): a randomised phase 3 trial. Lancet 2011;378:1931-9.

10. Bex A. Sequencing therapy for advanced renal cancer. Lancet Oncol 2020;21:9-11.

11. Motzer RJ, Escudier B, Tomczak P, et al. Axitinib versus sorafenib as second-line treatment for advanced renal cell carcinoma: overall survival analysis and updated results from a randomised phase 3 trial. Lancet Oncol 2013;14:552-62.

12. Choueiri TK, Escudier B, Powles T, et al. Cabozantinib versus everolimus in advanced renal-cell carcinoma. N Engl J Med 2015;373:1814-23.

13. Motzer RJ, Escudier B, McDermott DF, et al. Nivolumab versus everolimus in advanced renal-cell carcinoma. $\mathrm{N}$ Engl J Med 2015;373:1803-13.

14. Choueiri TK, Escudier B, Powles T, et al. Cabozantinib versus everolimus in advanced renal cell carcinoma (METEOR): final results from a randomised, open-label, phase 3 trial. Lancet Oncol 2016;17:917-27.

15. Escudier B, Sharma P, McDermott DF, et al. CheckMate
025 Randomized phase 3 study: outcomes by key baseline factors and prior therapy for nivolumab versus everolimus in advanced renal cell carcinoma. Eur Urol 2017;72:962-71.

16. Hotte SJ, Bjarnason GA, Heng DY, et al. Progression-free survival as a clinical trial endpoint in advanced renal cell carcinoma. Curr Oncol 2011;18 Suppl 2:S11-9.

17. Escudier B, Sharma P, McDermott DF, et al. Erratum to "CheckMate 025 Randomized Phase 3 Study: Outcomes by Key Baseline Factors and Prior Therapy for Nivolumab Versus Everolimus in Advanced Renal Cell Carcinoma" [Eur Urol 2017;72:962-71]. Eur Urol 2018;73:e116-8.

18. Heng DY, Xie W, Bjarnason GA, et al. Progression-free survival as a predictor of overall survival in metastatic renal cell carcinoma treated with contemporary targeted therapy. Cancer 2011;117:2637-42.

19. AVEO Oncology Announces Regulatory Update for Tivozanib in Renal Cell Carcinoma. AVEO. 2019. Available online: https://www.businesswire.com/news/ home/20191104005273/en/AVEO-Oncology-AnnouncesRegulatory-Update-Tivozanib-Renal

20. Aspinall SL, Zhao X, Geraci MC, et al. Use of targeted therapies for advanced renal cell carcinoma in the Veterans Health Administration. Cancer Med 2019;8:6651-61.
Cite this article as: Westerman ME, Wood CG. Editorial Commentary: Tivozanib versus sorafenib in patients with advanced renal cell carcinoma (TIVO-3): a phase 3, multicentre, randomised, controlled, open-label study. Ann Transl Med 2020;8(16):1037. doi: 10.21037/atm.2020.03.217 